\title{
Extension of the Burgess-Tully method to fit neutral atom and molecule cross-sections
}

\author{
H.P. Summers ${ }^{1}$ and T. Kato ${ }^{2}$ \\ 1 University of Strathclyde, 107 Rottenrow, Glasgow G4 0NG, UK \\ 2 National Institute for Fusion Science, Toki, Gifu, 509-5292, Japan
}

Received February 15; accepted March 27, 2000

\begin{abstract}
The method introduced by Burgess \& Tully (1992) for the examination and compacting of collision cross-section data for ions is extended to neutral atoms and molecules. To give adequate weighting and critical focus to the threshold region, as Burgess \& Tully did for the high energy asymptotic region, the energy and collision strength transformations have been modified. The new mapping is to the interval $[-1,1]$, with the excitation threshold region up to some switching energy mapped to $[-1,0]$. The high energy asymptotic region above the switching energy is mapped to the interval $[0,1]$. A ninepoint spline of equally spaced knots replaces the BurgessTully five-point spline. Appropriate threshold functional forms are incorporated into the description and the whole examination and fitting procedure is implemented as an interactive graphical utility under IDL that is available as a "stand-alone" code.
\end{abstract}

Key words: atomic data — spline interpolation

\section{Introduction}

For electron impact with ions, the collision strength is finite at threshold and in the high energy asymptotic limit it displays logarithmic, constant or inverse square behaviour depending on whether the transition is of dipole, nondipole, non-spin change or spin change type. There are also intermediate cases of small oscillator strength. It was pointed out by Burgess \& Tully (1992) that, with the emphasis on large collision calculations, such as those using the R-matrix method, and with the focus on resonance structure, attention was often not given to ensuring the correct continuation of the collision strength from low energies into the Born and Born/Ochkur regime at high energy. While this did not matter for forbidden line

Send offprint requests to: H.P. Summers formation in nebulae, it certainly did for fusion plasma applications. In the latter case, inflowing impurity species can find themselves in temperature regimes spanning from well below the ionisation energy to far above it. The high energy behaviour is of course simply prescribed, given the atomic structure, in terms of oscillator strength or first Born coefficients. Properly, the whole energy range of collision strength data should be provided for applications and the Burgess-Tully method of mapping the energy from threshold to infinity onto $[0,1]$ emphasised this. With the finite behaviour at threshold, the scaled collision strength for ions, as a function of scaled energy, displayed small variation over the whole independent variable range such that it could be well represented by a simple five-point equally-spaced knot spline. A single parameter, $C$, allowed a scale distortion in the independent variable by which the spline could be optimised. The plots became known as " $C$-plots" and often showed up embarrassing deficiencies in the original data.

With the introduction of divertor technology into magnetically confined fusion plasma design and the resurgence of discharge devices for plasma processing, neutral atoms and molecules are now very much the focus of interest in the laboratory environment (Behringer \& Fantz 1996) as well as of continuing importance in astrophysics (see, for example, the recent first observation of the neutral helium intercombination line in the solar atmosphere by Brooks et al. 1999). Collision strengths for neutrals are zero at threshold, with the behaviour near threshold dependent on the dominant partial wave in the $N+1$ electron system. Also, collision data is usually presented on scales which conceal the threshold region. The problem is analogous to that for the high energy region and a similar opening out of the threshold region as Burgess \& Tully did for the high energy region is desirable. There is a second issue. The Burgess-Tully method is not a faithful representation of the original data but a considered (and hopefully) improved conversion of the data. A second path of fitting data is that of strict interpolation on the assumption that 
the source data is exact. For this it is helpful to prepare an analytic approximate form $\Omega^{\text {(approx) }}$ which can be compared with the tabulated collision strength $\Omega$. It is useful if optimised fitting and interpolative fitting run side by side. Within the Atomic Data and Analysis Structure (ADAS) Project, established in support of the SOHO spacecraft and solar astrophysics (Mason et al. 1997; Summers et al. 1998; Summers 1993, 1999), both methods are used with the latter designed also to highlight misprints and errors of data entry.

\section{The transformations}

We note that the coordinate transformations used in the Burgess \& Tully method have a close connection with the general mathematical shape of the underlying collision strengths, so some commonality of the optimised fitting and strict interpolation approaches can be promoted by making the coordinate transformations and the approximate forms analytically similar. We shall continue to use the Burgess \& Tully transformations in this work and their distinction of collision strength types by a number $(1 \equiv$ dipole; $2 \equiv$ non-dipole, non-spin change; $3 \equiv$ spin change; 4 三 weak dipole) but they will apply strictly to the high energy region. We introduce new transformations for the threshold region and these will be distinguished by a letter. The transformations must satisfy certain monotonicity and continuity constraints - the latter at a switching energy where the low energy and high energy transformations meet. Then we require related approximate forms for the low and high energy regions which can again match smoothly at a switching energy.

The shapes of the energy transformations and approximate forms for the mapping and representation of collision strengths in the low energy (threshold) and high energy (asymptotic) regions which have proved useful and relevant to our further discussion are illustrated in Fig. 1. The dashed lines relate to transformations and the solid lines to approximate forms. The dashed curve (a'), with $B \sim 0.5$ ( $B$ is the threshold power law - cf. Eq. (1)) is the simplest monotonic form for the threshold behaviour of swave dominated collision strengths. Its curvature allows gradient matching with the energy transformation of logarithmic character for dipole allowed collision strengths of the Burgess-Tully type 1 and it is represented here by the solid line asymptotic form labelled 1 . For $B \geq 1.0$, the positive curvature is unsuitable for matching. The dashed curve (a) is also monotonic with the same threshold power law and is compatible with the energy transformations used in all of the Burgess-Tully types. The characteristic of non-dipole and spin-change collision strengths in the asymptotic region (solid lines (2)and (3) respectively) is to show negative gradients and the solid line threshold form (a) with $B=1.5$ is provided for matching in this case. The energy threshold transformation, dashed line (b), is an alternative to (a) but with an exponential character. We prescribe these transformations in detail below and the approximate forms in Sect. 4.

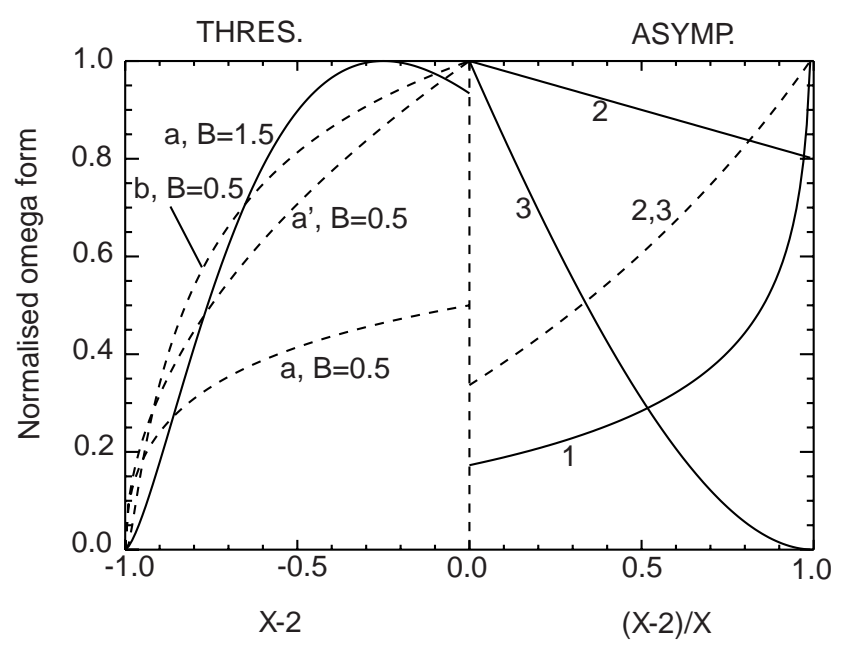

Fig. 1. The interval $[-1,0]$ shows threshold behaviours suitable for energy transformations (dashed) and approximate forms (solid), labelled by letter and parameter $B$ (see Sect. 2). The interval $[0,1]$ shows similarly the asymptotic transformations and forms, labelled by numerals. The curves are unmatched between the threshold and asymptotic regions only because they have been normalised to unit peak value and/or adjusted to emphasis the shapes. $X$ is the threshold scaled energy parameter. The labelling follows the classification scheme given in Sect. 2

Let the transition energy between lower level $i$ and upper level $f$ be $E_{i f}$. The threshold scaled energy parameter is defined by $X=\left(E_{f} / E_{i f}\right)+1$. Working in energies $E_{f}$ relative to the threshold energy, let $E_{0}$ mark the division between the threshold region and the high energy region. We call this the switching energy. We seek mappings $x_{-}$ and $x_{+}$of the independent variable from final energies $\left[0, E_{0}\right]$ to $[-1,0]$ and from final energies $\left[E_{0}, \infty\right]$ to $[0,1]$ respectively and compatible transformations of the collision strengths. We introduce a scale parameter, $B$, for the threshold region and $C$, for the high energy region. $C$ is the Burgess-Tully parameter. The transformations are given below, indexed by Burgess-Tully type (see Burgess \& Tully 1992) and a letter for the threshold type.

\subsection{Type $1 a$}

$$
\begin{aligned}
x_{+}= & 1-\ln (C) / \ln \left(\left(E_{f}-E_{0}\right) / E_{i f}+C\right) \\
x_{-}= & 2\left(E_{f} / E_{0}\right)^{B} /\left(\left(E_{f} / E_{0}\right)^{B}+1\right)-1 \\
y_{+}= & \Omega / \ln \left(\left(E_{f}-E_{0}\right) / E_{i f}+\mathrm{e}\right) \\
y_{-}= & \Omega /\left(( E _ { f } / E _ { 0 } ) ^ { B } \operatorname { e x p } \left(-\left(B-E_{0} / \mathrm{e} E_{i f}\right)\right.\right. \\
& \left.\left.\left(\left(E_{f} / E_{0}\right)-1\right)\right)\right) \\
y_{+}(0)= & \Omega\left(E_{0}\right)
\end{aligned}
$$


$y_{+}(1)=4 \omega_{i} f_{i f} / E_{i f}$

\subsection{Type $2 a$}

$$
\begin{aligned}
x_{+} & =\left(\left(E_{f}-E_{0}\right) / E_{i f}\right) /\left(\left(\left(E_{f}-E_{0}\right) / E_{i f}\right)+C\right) \\
x_{-} & =2\left(E_{f} / E_{0}\right)^{B} /\left(\left(E_{f} / E_{0}\right)^{B}+1\right)-1 \\
y_{+} & =\Omega \\
y_{-} & =\Omega /\left(\left(E_{f} / E_{0}\right)^{B} \exp \left(-B\left(\left(E_{f} / E_{0}\right)-1\right)\right)\right) \\
y_{+}(0) & =\Omega\left(E_{0}\right) .
\end{aligned}
$$

\subsection{Type $3 a$}

$$
\begin{aligned}
x_{+}= & \left(\left(E_{f}-E_{0}\right) / E_{i f}\right) /\left(\left(\left(E_{f}-E_{0}\right) / E_{i f}\right)+C\right) \\
x_{-}= & 2\left(E_{f} / E_{0}\right)^{B} /\left(\left(E_{f} / E_{0}\right)^{B}+1\right)-1 \\
y_{+}= & \left.\left(\left(E_{f}-E_{0}\right) / E_{i f}\right)+1\right)^{2} \Omega \\
y_{-}= & \Omega /\left(( E _ { f } / E _ { 0 } ) ^ { B } \operatorname { e x p } \left(-\left(B+2 E_{0} / E_{i f}\right)\right.\right. \\
& \left.\left.\left(\left(E_{f} / E_{0}\right)-1\right)\right)\right) \\
y_{+}(0)= & \Omega\left(E_{0}\right) .
\end{aligned}
$$

\subsection{Type $4 a$}

$$
\begin{aligned}
x_{+}= & 1-\ln (C) / \ln \left(\left(E_{f}-E_{0}\right) / E_{i f}+C\right) \\
x_{-}= & 2\left(E_{f} / E_{0}\right)^{B} /\left(\left(E_{f} / E_{0}\right)^{B}+1\right)-1 \\
y_{+}= & \Omega / \ln \left(\left(E_{f}-E_{0}\right) / E_{i f}+C\right) \\
y_{-}= & \Omega /\left(\ln (C)\left(E_{f} / E_{0}\right)^{B} \exp (\right. \\
& \left.\left.-\left(B-E_{0} / C \ln (C) E_{i f}\right)\left(\left(E_{f} / E_{0}\right)-1\right)\right)\right) \\
y_{+}(0)= & \Omega\left(E_{0}\right) / \ln (C) \\
y_{+}(1)= & 4 \omega_{i} f_{i f} / E_{i f} .
\end{aligned}
$$

An alternative transformation of the independent variable in the threshold region is also provided which is of exponential rather than power law type.

\subsection{Type $b$}

$x_{-}=\exp \left(-B\left[\sqrt{\left(E_{i f} / E_{f}\right)}-\sqrt{\left(E_{i f} / E_{0}\right)}\right]\right)-1$.

These transformations introduce three parameters, $B, C$, and $E_{0}$. We impose a requirement of continuity on the derivative of $x\left(E_{f}\right)$ at $x=0$ which determines $E_{0}$ in terms of $B$ and $C$. For example, for types $1 \mathrm{a}$ and $4 \mathrm{a}, E_{0}=$ $E_{i f} B C \ln (C) / 2$; for types $2 \mathrm{a}$ and $3 \mathrm{a}, E_{0}=E_{i f} B C / 2$; for types $1 \mathrm{~b}$ and $4 \mathrm{~b}, E_{0}=E_{i f}(B C \ln (C) / 2)^{2 / 3}$; for types $2 \mathrm{~b}$ and $3 \mathrm{~b}, E_{0}=E_{i f}(B C / 2)^{2 / 3}$. Thus the Burgess-Tully $C$ parameter allows the usual distortion of the high energy $x$ scale while the $B$ parameter allows stretching or contraction of the threshold region. Note that the transformations maintain continuity of value and derivative of $x_{-}$and $x_{+}$ at $E_{f}=E_{0}$ and also of $y_{-}$and $y_{+}$. The stretching enabled by the variation of $B$ and $C$ allows convergence on the best matching and fit of the threshold and asymptotic regions.

\section{A nine-point least squares spline}

In the spirit of Burgess \& Tully, it is appropriate to seek an optimised least squares spline to the data and that this should now be based on the nine fixed knot positions $\left[x_{i}=i / 4: i=-4, \ldots,+4\right]$. The spline is specified with constant curvature at both ends. The spline evaluation routine takes as input the $x$ value and the spline values at the nine knot positions. The least squares algorithm for optimally determining the knot values follows the same pattern as that of Burgess \& Tully. The special cases of under-determined splines, that is when the number of data values $N \leq 9$ were tedious to prepare but use Lagrangian inverse interpolation to reduce progressively the number of active knots to match the number of data values. Figure 2 a shows the $B-C$ plot for electron-impact excitation of He I $1 s^{2}{ }^{1} \mathrm{~S}-2 \mathrm{~s} 2 \mathrm{p}{ }^{1} \mathrm{P}$. The data are from the assessment by de Heer et al. (1992) and have been analysed as type 1a. Figure 3 a shows the He I $1 \mathrm{~s}^{2}{ }^{1} \mathrm{~S}-$ $2 \mathrm{~s} 2 \mathrm{p}{ }^{3} \mathrm{P}$ electron impact excitation from the same source, analysed as type 3 a.

\section{Interpolation}

For the strict interpolation approach, we note firstly that, if a good approximate form $\Omega^{\text {(approx) }}$, representing the collision strength $\Omega$, is available, then a graph of the ratio $\left[\Omega\left(X_{i}\right) / \Omega_{i}^{\text {(approx) }}: i=1, \ldots, N\right]$ draws attention clearly to errors or misprints in the tabular source data. Also, the interpolating spline through the ratio values (which will then not differ substantially from unity) is accurate. We seek to introduce good approximations to collision strengths based on the following parametrised approximate forms. The approximate forms for neutral species have two parts $(-$ and +$)$ which apply to the threshold and asymptotic regions respectively and the two parts are matched at some tabular value of the collision parameter $X=X_{k}$ :

\subsection{Type $1 a$}

$$
\begin{aligned}
\Omega_{+}^{(\text {approx })}= & F_{3} S \ln \left(X-X_{k}+F_{2}\right) \\
\Omega_{-}^{(\text {approx })}= & F_{1}\left((X-1) /\left(X_{k}-1\right)\right)^{B} \exp \left(-\left(B-b^{\prime}\right)\right. \\
& \left.\left(\left(X-X_{k}\right) /\left(X_{k}-1\right)\right)\right)
\end{aligned}
$$

where $b^{\prime}=\left(X_{k}-1\right) F_{3} S / F_{1} F_{2}$ and $F_{1}=\Omega_{k}$.

\subsection{Type $2 a$}

$$
\begin{aligned}
\Omega_{+}^{(\text {approx })}= & F_{2}+F_{3} /\left(X-X_{k}+1\right) \\
\Omega_{-}^{(\text {approx })}= & F_{1}\left((X-1) /\left(X_{k}-1\right)\right)^{B} \exp \left(-\left(B-b^{\prime}\right)\right. \\
& \left.\left(\left(X-X_{k}\right) /\left(X_{k}-1\right)\right)\right)
\end{aligned}
$$

where $b^{\prime}=\left(X_{k}-1\right) F_{3} / F_{1}$ and $F_{1}=\Omega_{k}$. 

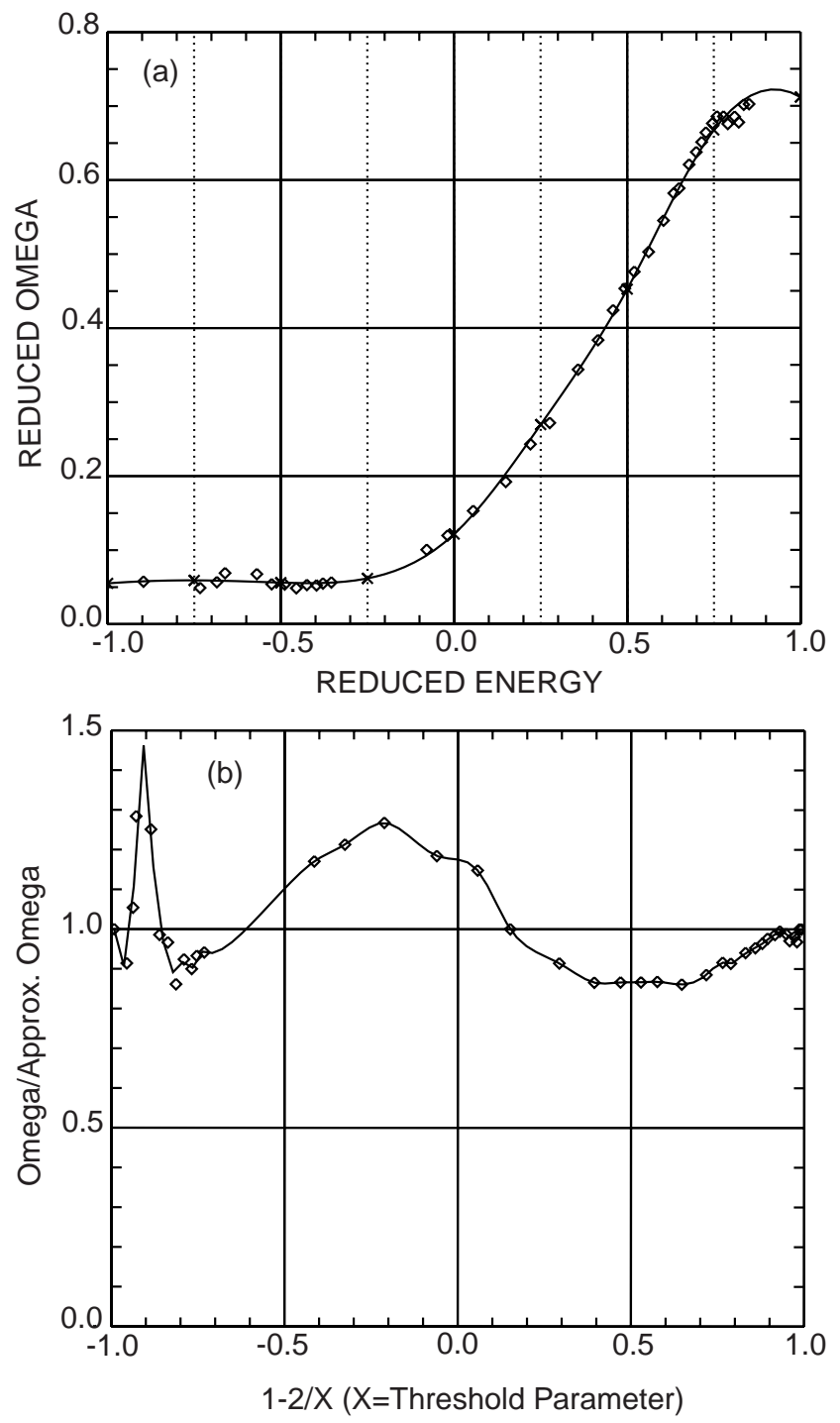

Fig. 2. a) Type 1a: $B-C$ nine-knot spline least squares fit to the $\operatorname{HeI}\left({ }^{1}{ }^{1} \mathrm{~S}-2{ }^{1} \mathrm{P}\right)$ dipole-allowed electron-impact collision strength data of de Heer et al. (1992). $B=0.593$, $C=2.251$; b) Type $1 \mathrm{a}: \Omega / \Omega^{\text {(approx) }}$ ratio plot and interpolating spline for the same dipole-allowed collision strength data. $B=0.53828, b^{\prime}=1.70840, F_{1}=0.34300, F_{2}=1.62850, F_{3}=$ $1.32310, X_{k}=2.35670$. Note the variability of the tabular data which gives grounds for adjustment of some values. The stated uncertainty of the data is $\sim 10 \%$

\subsection{Type $3 a$}

$$
\begin{aligned}
\Omega_{+}^{(\text {approx })}= & F_{3} /\left(X-X_{k}+F_{2}\right)^{2} \\
\Omega_{-}^{(\text {approx })}= & F_{1}\left((X-1) /\left(X_{k}-1\right)\right)^{B} \exp \left(-\left(B-b^{\prime}\right)\right. \\
& \left.\left(\left(X-X_{k}\right) /\left(X_{k}-1\right)\right)\right)
\end{aligned}
$$

where $b^{\prime}=-2\left(X_{k}-1\right) F_{3} / F_{1} F_{2}^{3}$ and $F_{1}=\Omega_{k}$.

\subsection{Type $4 a$}

$\Omega_{+}^{(\text {approx })}=1$

$\Omega_{-}^{(\text {approx })}=1$.

We note the inclusion of the line strength in the definition of type 1 . If high energy data is correct and consistent with the line strength then $F_{3}$ should be equal to $4 / 3$. Burgess \& Tully impose this condition on their optimised fit. Type 4 is the case of no approximate form. There is no adjustable transformation of the independent variable $X$ then but, as in the Burgess-Tully case, it is convenient to transform to $X^{\prime}=1-2 / X$ for displaying the ratio over the finite range $[-1,1]$ with a balanced weighting between the threshold and asymptotic regions. The parameters of the approximate forms are determined by fixing $\Omega_{-}^{\text {(approx) }}\left(X_{0}\right)=\Omega_{0}, \Omega_{+}^{\text {(approx) }}\left(X_{N}\right)=\Omega_{N}$ and $\Omega_{+}^{\text {(approx) }}\left(X_{k}\right)=\Omega_{-}^{\text {(approx) }}\left(X_{k}\right)=\Omega_{k}$. Note that the $\Omega$ values at $X_{N}$ and $X_{k}$ fix $F_{2}$ and $F_{3}$, explicitly for types 2 a and $3 \mathrm{a}$ and implicitly for type $1 \mathrm{a}$. Then $b^{\prime}$ is determined explicitly from continuity of the derivative at $X_{k}$, that is $\Omega_{+}^{{\text {(approx })^{\prime}}^{\prime}}\left(X_{k}\right)=\Omega_{-}^{\text {(approx) }}{ }^{\prime}\left(X_{k}\right)$, so that an implicit equation is left finally for determining $B$. The procedure requires $N \geq 3$. For $N=2$, we force $F_{3}=4 / 3$ for type $1, F_{3}=0$ for type 2 and $F_{2}=1$ for type 3 . For $N=1$, we force $B=0.5$. Also, data which do not manifest the expected monotonic asymptotic behaviours can prevent solution for the approximate form. The forced contractions given above are again imposed in such cases. For historical reasons, in the ADAS graphical user interface, the normal solution for the approximate form is called "2point" fitting and the contracted problem cases, "1-point" fitting.

For $N \geq 4$, there is a choice of matching position. We seek $X_{k}$ with $2 \leq k \leq N-1$ so that $\sum_{i=1}^{N}\left(\Omega\left(X_{i}\right)-\right.$ $\left.\Omega^{\text {(approx) }}\left(X_{i}\right)\right)^{2}$ is a minimum. If $k=1$, the tabular data set does not span the threshold region effectively and so we set $B=0.5$. If $k=N$, the tabular data set does not span the high energy region effectively and so we set $F_{3}=(4 / 3)$. Figures $2 \mathrm{~b}$ and $3 \mathrm{~b}$ show the interpolation of the $\Omega / \Omega^{\text {(approx) }}$ ratio graph. The data are for the same examples as in Figs. 2a and 3a respectively. The cases are again analysed as types $1 \mathrm{a}$ and $3 \mathrm{a}$.

Figures 4 a-c show the $\Omega / \Omega^{\text {(approx) }}$ ratio and $B-C$ plots for the spin change hydrogen molecular reaction crosssection $H_{2}\left(X^{1} \Sigma_{g}^{+}\right)+\mathrm{e} \rightarrow H_{2}\left(d^{3} \Pi_{u}^{+}\right)+\mathrm{e}$. The source data are from Behringer \& Fantz (1996) and are themselves a fit to very limited experimental data (Mohlmann \& De Heer 1976). The ratio plot seems to indicate that the first data value is too low, while the last two data values suggest that there is some deviation (again too low) from the expected high energy behaviour. The $B-C$ plot for these data (Fig. 4b) shows that the spline diverges at both limits for reasonable values of the parameters. In Fig. 4c, the first and last two data points have been adjusted (see 

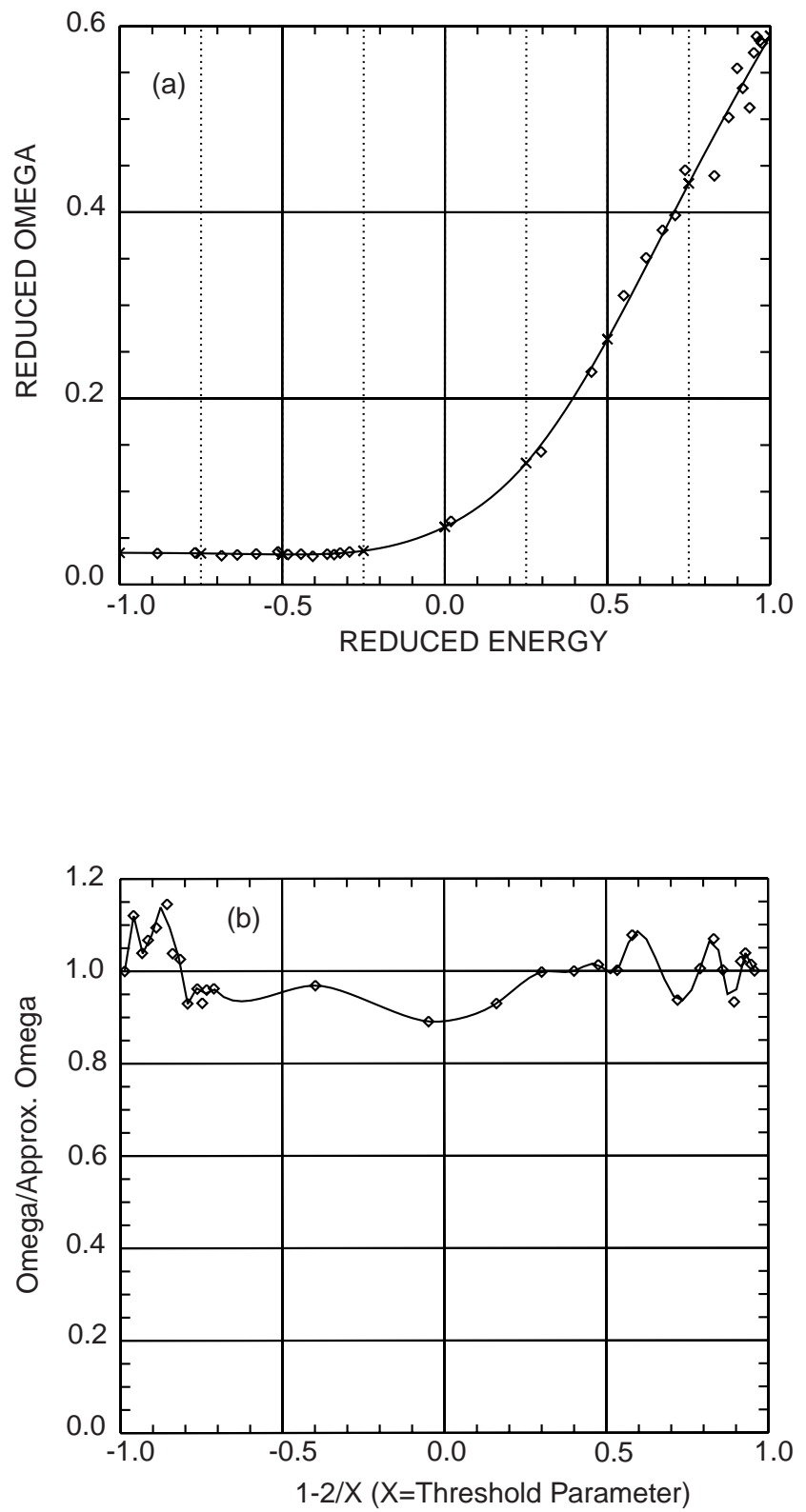

Fig. 3. a) Type $3 a: B-C$ nine-knot spline least squares fit to the $\operatorname{HeI}\left(1{ }^{1} \mathrm{~S}-2{ }^{3} \mathrm{P}\right)$ spin-change electron-impact collision strength data of de Heer et al. (1992). $B=0.685, C=1.191$; b) Type 3a: $\Omega / \Omega^{\text {(approx) }}$ ratio plot and interpolating spline for the same spin-change collision strength data. $B=0.56483$, $b^{\prime}=-1.21690, F_{1}=0.04090, F_{2}=3.84420, F_{3}=0.60442$, $X_{k}=3.33900$. The stated uncertainty of the data is $\sim 10 \%$

Sect. 6 below) and an optimised fit then obtained. These adjustments are well outside the range of the original experimental data and perhaps deliver a safer extrapolation in the limiting asymptotic and threshold regions.
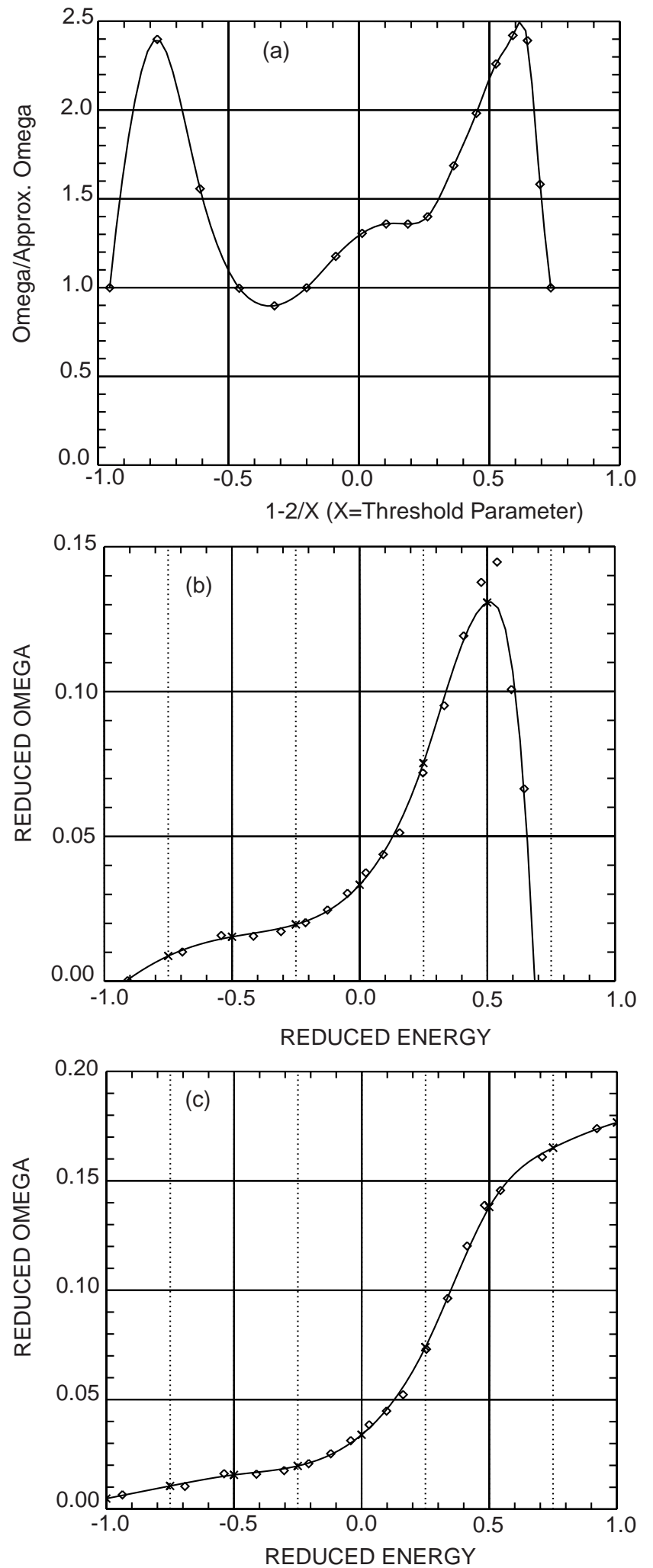

Fig. 4. $\mathrm{H}_{2}\left(X^{1} \Sigma_{g}^{+}\right)+\mathrm{e} \rightarrow \mathrm{H}_{2}\left(d^{3} \Pi_{u}^{+}\right)+$e. a) Type 3a interpolative ratio plot, $B=2.65490, b^{\prime}=-1.01780, F_{1}=0.04910$, $\left.F_{2}=1.30870, F_{3}=0.08409, X_{k}=1.66600 ; \mathbf{b}\right) B-C$ plot with original data, $B=0.778, C=2.982 ;$ c) $B-C$ plot after adjustment of first and last two data values, $B=0.778, C=2.940$ 


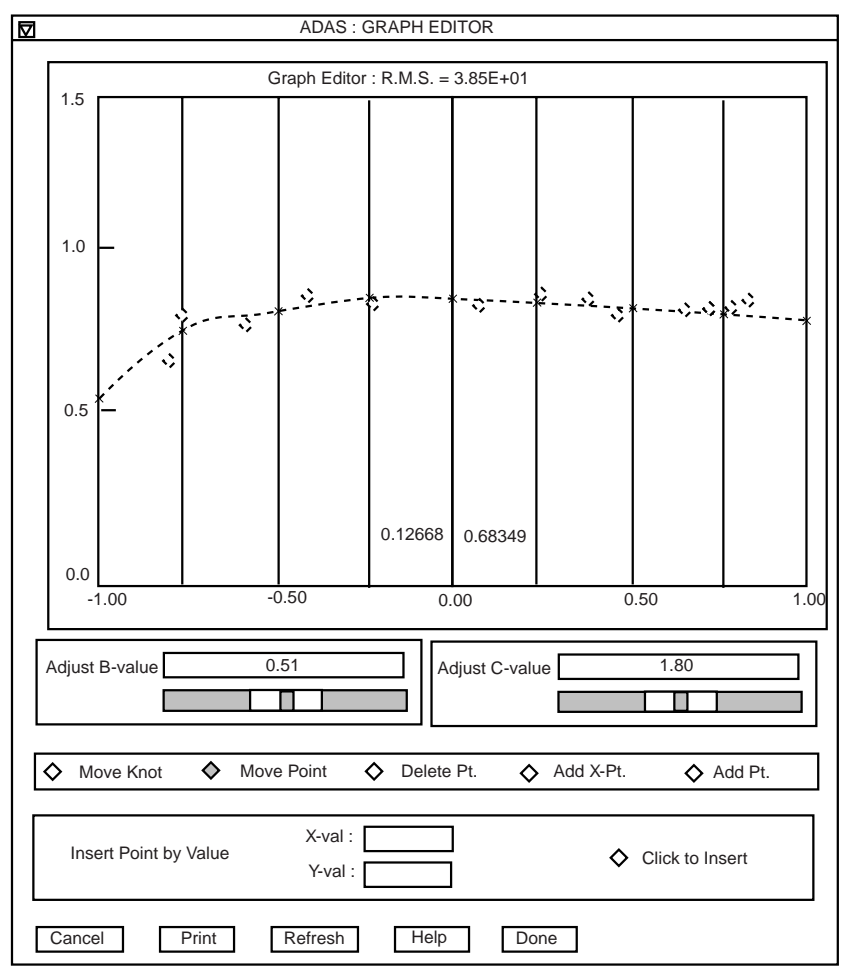

Fig. 5. An editable graph window with an active $B-C$ plot. The number pair at the bottom of the displayed graph shows the cursor position, in graph coordinates, when a point is being moved

\section{Quadratures}

The production of Maxwell-averaged collision strengths is straightforward. The Burgess-Tully Gauss-Laguerre based approach remains valid for the high energy region. The finite range of the low-energy region is treated similarly but with the Gaussian quadrature, in this case tuned to the usual $\sqrt{X-1}$ behaviour. The switch in energy between the two quadratures is not set at the $E_{0}$ of Sect. 2 or the $X_{k}$ of Sect. 4 but, rather, where the threshold collision strength and Maxwellian exponential energy-scale lengths are equal. For the strict interpolative approach, it is noted that the approximate forms are integrable in terms of known functions. Thus, setting $a_{\mathrm{T}}=\left(E_{i f} / I_{\mathrm{H}}\right)\left(I_{\mathrm{H}} / k T_{\mathrm{e}}\right)$ with $I_{\mathrm{H}}$ the Rydbery energy unit, the Maxwell averaged collision strength is

$\Upsilon^{\text {(approx })}\left(T_{\mathrm{e}}\right)=\Upsilon_{-}^{\text {(approx })}\left(T_{\mathrm{e}}\right)+\Upsilon_{+}^{(\text {approx })}\left(T_{\mathrm{e}}\right)$.

\subsection{Type $1 a$}

$$
\begin{aligned}
\Upsilon_{-}^{\text {(approx })}\left(T_{\mathrm{e}}\right)= & F_{1}\left(X_{k}-1\right) a_{\mathrm{T}} \\
& \mathrm{e}^{B-b^{\prime}} \frac{1}{|A|^{B+1}} \gamma(B+1, A) \\
\Upsilon_{+}^{\text {(approx })}\left(T_{\mathrm{e}}\right)= & \mathrm{e}^{-\left(X_{k}-1\right) a_{\mathrm{T}}} F_{3} S_{i f} \\
& {\left[\ln \left(F_{2}\right)+\mathrm{e}^{a_{\mathrm{T}} F_{2}} E_{1}\left(a_{\mathrm{T}} F_{2}\right)\right] }
\end{aligned}
$$

\section{Implementation as an IDL/ADAS code}

Codes that are available within the ADAS Project (Summers 1993, 1999) use IDL as the graphical user interface and FORTRAN for calculations. Each code uses a basic three window sequence composed of a file (or archive) selection window, a user data entry window and finally a graphical display and output routing window. Only the graphical display part, where the actual collision strength fitting takes place, is of interest to the non-ADAS user and this is available as a self-contained single IDL utility with simplified data inputs and outputs. The graphic display window is a variant of the ADAS Graph Editor developed by Brooks (1997) and has the appearance shown in Fig. 5 .

In the $B-C$ plot mode, there are two sliders below the graph which control the $B$ and $C$ parameters. The spline is 
continually updated as the sliders are moved. The graph is editable, in that both the data values and the spline knot values can be modified. Operation is by a mouse click on the appropriate operation button and then dragging (or clicking on) a data point on the screen with the left mouse button. Clicking the right mouse button in the screen area terminates the operation. Multiple data points may be operated on before termination. Movement (Move Point) or deletion (Delete Pt.) of data points leads to recycling back to the graph editor before output. The $B-C$ plot spline knot values can only be moved vertically (Move Knot) since the knot positions are fixed. New data points can be added between existing data points by single mouse click, when this is unambiguous ( $A d d X$-Pt.). A point can be added anywhere by two mouse clicks - the first identifies an existing point in the set of values which the new point is to follow ( $A d d P t$.).

In interpolation mode, the sliders are not present, but the other operations are as above.

\section{Conclusions}

The threshold behaviour of neutral atom and molecule collision strengths is not as clearly or simply determined as the high energy behaviour, with out access to detailed cross-section calculations. Thus the present extension of the Burgess-Tully method should be viewed as less rigorous than the original work and more as an operational convenience. Extrapolation and interpolation of collision data remain problematic in the case of very few data values and there is no real guarantee of precision. In practical terms, the $B-C$ spline becomes markedly sensitive to small variations of the parameters when there are few data values, but then the strict interpolative approach based on approximate forms is both stable and safe.
The method can be applied without change to electron impact ionisation collision strengths. However, it has been the practice to fit ionisation collision strengths by synthesising a superposition of direct and autoionisation components with adjustable threshold energies and equivalent electron multipliers. The latter approach is philosophically incompatible with the present method.

Acknowledgements. One of us (HPS) wishes to thank the National Institute of Fusion Science in Japan for their kind sponsorship of a visiting position. Dr. D. Brooks and Dr. A. Lanzafame prepared the ADAS Fortran version of the original Burgess-Tully C-plot code for ions.

\section{References}

Behringer K.H., Fantz U., 1996 (private communication)

Brooks D.H., Fischbacher G.A., Fludra A., et al., 1999, A\&A 347,277

Brooks D.H., 1997, Ph.D. Thesis, University of Strathclyde

Burgess A., Tully J.A., 1992, A\&A 254, 436

de Heer F.J., Hoekstra R., Kingston A.E., Summers H.P., 1992, Nucl. Fusion Suppl. 3, 19

Mason H.E., Young P.R., Pike C.D., et al., 1997, "The First Results from SOHO" Fleck B \& Svestka Z. (eds.). Kluwer Academic Publishers, The Netherlands, pp. 143-161

Mohlmann G.R., De Heer F.J., 1976, Chem. Phys. Lett. 43, 240

Summers H.P., 1993, JET Joint Undertaking Report JET$\operatorname{IR}(93) 07$

Summers H.P., 1999, The ADAS User Manual, version 2.1 http://patiala.phys.strath.ac.uk/adas/

Summers H.P., Anderson A., Badnell N.R., et al., 1998, "Atomic and Molecular Data and Their Applications", Mohr P.J. and Wiese W.L. (eds.). American Institute of Physics Conf. Proc. 434, pp. 259-286 